\title{
Efficiency of off-axis astronomical adaptive systems: Comparison of theoretical and experimental data
}

\author{
V.V. Voitsekhovich ${ }^{1}$, V.G. Orlov ${ }^{1}$, S. Cuevas ${ }^{1}$, and R. Avila ${ }^{2}$ \\ 1 Instituto de Astronomía, UNAM AP 70-264 Cd. Universitaria, 04510 Mexico D.F., Mexico \\ 2 Département d'Astrophysique UMRA 6525, Université de Nice-Sophia Antipolis CNRS, Parc Valrose, 06108 Nice Cedex 2 , \\ France
}

Received March 30; accepted June 30, 1998

\begin{abstract}
The efficiency of off-axis adaptive astronomical systems is estimated using both the theoretical Hufnagel model and recently obtained experimental $C_{n}^{2}$ profiles. The efficiency of interest is considered through the Strehl ratio of the corrected image. The experimental $C_{n}^{2}$ profile used in calculations is a result of measurements carried out at the Observatorio Astronomica Nacional at San Pedro Mártir (Mexico). It is found that there is a significant difference in the efficiency calculated from both profiles because they differ strongly in structure. The experimental one has a pronounced layered structure with a great amount of the turbulence strength concentrated near the ground, while the theoretical profile suggests more smooth behavior of $C_{n}^{2}$ with altitude.
\end{abstract}

Key words: atmospheric effects - telescope instrumentation: adaptive optics

\section{Introduction}

The concept of off-axis adaptive astronomical systems has become quite popular in recent years because it gives a principal possibility to override certain difficulties arising when the real-time correction has to be applied for weak stars (or for extended objects). This approach assumes that some star separated by a small angle from the weak star of interest is used as a reference one. If the reference star is bright enough to perform real-time wavefront measurements, the information obtained can be used for improvement of the weak star image. However in order to get an improvement, the separation angle has to be chosen in such a way that the wavefront distortions associated with both stars are correlated; otherwise the effect will be negative. On the other hand, one needs some criterion to estimate a resulting quality of correction. The long-exposure

Send offprint requests to: V.G. Orlov
Strehl ratio to be obtained for the weak star after correction is an observable and meaningful criterion, so it is of practical interest to calculate this parameter as a function of the separation angle, propagation and turbulence conditions, adaptive system performances, etc.

In this paper we restrict our consideration to the case of perfect adaptive correction that allows us to concentrate the attention on some principal aspects of off-axis correction and on the calculations with recently obtained experimental data. The problem under treatment is a part of more general one: the so-called isoplanatic problem. As for the theoretical development related to the last problem, many approaches based on different considerations were suggested (Wang 1975; Shapiro 1976; Fried 1978, 1982; Roddier 1981; Chassat 1989; Welsh \& Gardner 1991; Sasiela \& Shelton 1993). However, as for the weakturbulence conditions that is the case for astronomical applications, one can get the straightforward solution. The result of interest follows directly if one applies the Rytov method for solution of the parabolic equation (Tatarski 1968) which describes the wave propagation through the atmospheric turbulence. We refer and outline this approach in Sect. 1. Based on this solution, we calculate the residual structure function of the phase fluctuations associated with the on-axis star. Then we introduce an isotropic approximation for this function that simplifies significantly the calculation of Strehl ratio. In Sect. 2, we present the results of the calculations for the Strehl ratio with both the theoretical model of $C_{n}^{2}$ profile and with the experimental profile recently measured in San Pedro Mártir (SPM) observatory (Mexico). The results obtained are compared and discussed at the end of that section.

\section{Theoretical treatment}

The propagation of light waves through the turbulent atmosphere is described by the so-called parabolic partial differential equation (Tatarski 1968). For the 
weak-turbulence conditions which take place under astronomical observations, this equation can be solved by the Rytov method (Tatarski 1968) which is actually the conventional perturbation method but applied to the logarithm of the field rather than to the field itself. If one places the $X$ and $Y$ axes of the three-dimensional Cartesian coordinate system at the aperture plane and considers the initial monochromatic plane wave propagating through the turbulence along the $Z$-axis, the classic Rytov solution is given by Tatarski (1968):

$$
\begin{aligned}
\Psi_{1}(\boldsymbol{\rho})= & \chi_{1}(\boldsymbol{\rho})+i S_{1}(\boldsymbol{\rho})= \\
& i k \int_{0}^{L} \mathrm{~d} z \int \mathrm{d}^{2} \kappa g_{n}(z, \boldsymbol{\kappa}) \exp \left(i \boldsymbol{\kappa} \cdot \boldsymbol{\rho}+\frac{\kappa^{2} z}{2 i k}\right),
\end{aligned}
$$

where $\chi_{1}$ and $S_{1}$ denote the log-amplitude and the phase fluctuations in the aperture plane, respectively, $k$ is the wavenumber, $L$ is the propagation length, $g_{n}$ is the spectrum of fluctuations of the refractive-index random field, and $\boldsymbol{\rho}$ denotes the two-dimensional vector at the aperture plane.

The Rytov solution for the off-axis propagation was obtained in Vitrichenko et al. (1984). It follows straightforwardly if one applies the same Rytov method, but for the initially tilted plane wave. Assuming that this wave has the propagation unit vector $\boldsymbol{n}=(\cos \alpha, \cos \beta, \cos \gamma)$, where $\alpha, \beta$, and $\gamma$ are the angles between the vector $\boldsymbol{n}$ and the axes $X, Y$, and $Z$, respectively, one can get (Vitrichenko et al. 1984)

$\Psi_{2}(\boldsymbol{\rho})=$

$i k \int_{0}^{L} \mathrm{~d} z \int \mathrm{d}^{2} \boldsymbol{\kappa} g_{n}(z, \boldsymbol{\kappa}) \exp \left(i \boldsymbol{\kappa} \cdot \boldsymbol{\rho}+\frac{\kappa^{2} z}{2 i k}-i \boldsymbol{\kappa} \cdot \boldsymbol{n}_{\perp} z\right)$,

$\boldsymbol{n}_{\perp}=(\sin \gamma \cos \alpha, \sin \gamma \cos \beta)$.

The derivation of Eq. (2) is practically the same as for the along-axis propagation, so we do not describe it here referring the reader to Tatarski (1968) for details. The only difference is that the tilted wave is considered as the initial condition for the Rytov solution of parabolic equation.

Equations $(1,2)$ are sufficient to calculate main statistical quantities which are of interest in isoplanatic-related problems. Let us apply these results to our problem.

Let $\gamma$ be the separation angle between the two stars (1 and 2), observed through the atmosphere. To simplify the mathematical derivations, we assume that the star 1 is on the telescope axis, but the generalization of results for the off-axis location of the star 1 is straightforward. Then, let us suppose that the phase fluctuations $S_{2}$ are perfectly measured and that this measurement is used as the compensation signal to perform the adaptive correction for the star 1 . We are interested in calculation of the long-exposure Strehl ratio to be reached for the star 1 in the case of perfect phase measurement and correction. Under these conditions the residual phase distortion $S_{\mathrm{R}}$ of the star 1 is $S_{\mathrm{R}}=S_{1}-S_{2}$. The phase fluctuations $S_{1}$ and $S_{2}$ of the stars 1 and 2 at the aperture plane are determined from Eq. (1) and Eq. (2), respectively, and the associated residual structure function $D_{\mathrm{S}_{\mathrm{R}}}$ is expressed as

$$
\begin{array}{r}
D_{\mathrm{S}_{\mathrm{R}}}\left(\boldsymbol{\rho}_{1}, \boldsymbol{\rho}_{2}\right)=2.61 k^{2} \int_{0}^{L} \mathrm{~d} z C_{n}^{2}(z) \int \mathrm{d}^{2} \boldsymbol{\kappa}^{-11 / 3} \\
\times\left\{1-\exp \left[i \boldsymbol{\kappa} \cdot\left(\boldsymbol{\rho}_{1}-\boldsymbol{\rho}_{2}\right)\right]\right\}\left[1-\cos \left(\boldsymbol{\kappa} \cdot \boldsymbol{n}_{\perp} z\right)\right],
\end{array}
$$

where $C_{n}^{2}$ denotes the refractive-index structure characteristic, and the isotropic Kolmogorov spectrum $\Phi_{n}(\kappa, z)=$ $0.033 C_{n}^{2}(z) \kappa^{-11 / 3}$ of refractive-index fluctuations has been used. Also, we have used the approximated equality $1+\exp \left(\frac{\kappa^{2} z}{i k}\right) \approx 2$, which, as it has been shown in Tatarski (1968), holds with a high accuracy for the light wave propagation through the weak-turbulent atmosphere. A procedure of the statistical average for the quantities $g_{n}(z, \boldsymbol{\kappa})$ which has been applied to get Eq. (3) from Eqs. $(1,2)$ is described in details in Tatarski (1968). Actually, to perform this average, it is sufficient to know only two equalities:

$$
\begin{aligned}
& \left\langle g_{n}\left(z_{1}, \boldsymbol{\kappa}_{1}\right) g_{n}^{*}\left(z_{2}, \boldsymbol{\kappa}_{2}\right)\right\rangle \\
& =2 \pi \Phi_{n}\left(z_{1}, \boldsymbol{\kappa}_{1}\right) \delta\left(z_{1}-z_{2}\right) \delta\left(\boldsymbol{\kappa}_{1}-\boldsymbol{\kappa}_{2}\right), \\
& \left\langle g_{n}\left(z_{1}, \boldsymbol{\kappa}_{1}\right) g_{n}\left(z_{2}, \boldsymbol{\kappa}_{2}\right)\right\rangle \\
& =2 \pi \Phi_{n}\left(z_{1}, \boldsymbol{\kappa}_{1}\right) \delta\left(z_{1}-z_{2}\right) \delta\left(\boldsymbol{\kappa}_{1}+\boldsymbol{\kappa}_{2}\right),
\end{aligned}
$$

where the sign $(*)$ stands for the complex conjugation, $\delta$ denotes the Dirac delta-function, and $\Phi_{n}$ is the spectrum of the refractive-index fluctuations.

As one can see from Eq. $(3), D_{\mathrm{S}_{\mathrm{R}}}\left(\boldsymbol{\rho}_{1}, \boldsymbol{\rho}_{2}\right)$ is an anisotropic function that is not convenient for the calculation of the Strehl ratio. So, we calculate its isotropic approximation as follows. Introducing the polar coordinates at the aperture plane, we can express the scalar product $\boldsymbol{\kappa} \cdot \boldsymbol{n}_{\perp}$ as

$\boldsymbol{\kappa} \cdot \boldsymbol{n}_{\perp}=\kappa \sin \gamma \cos (\varphi-\theta)$,

where $\gamma$ is the separation angle between the stars, $\varphi$ is the polar angle of projection of the propagation vector $\boldsymbol{n}$ on the aperture plane, and $\theta$ is the polar angle of the vector $\boldsymbol{\kappa}$.

Substituting Eq. (4) into Eq. (3), averaging $D_{\mathrm{S}_{\mathrm{R}}}\left(\boldsymbol{\rho}_{1}, \boldsymbol{\rho}_{2}\right)$ over the angle $\varphi$, evaluating the integrals over $\theta$ and $\kappa$, and taking into account that for the most cases of practical concern $\sin \gamma \approx \gamma$, we get the following isotropic approximation for the residual structure function:

$$
\begin{gathered}
D_{\mathrm{S}_{\mathrm{R}}}\left(r=\left|\boldsymbol{\rho}_{1}-\boldsymbol{\rho}_{2}\right|\right)=5.83 k^{2} \int_{0}^{L} \mathrm{~d} z C_{n}^{2}(z) \\
\times\left\{r^{5 / 3}+(\gamma z)^{5 / 3}\right. \\
\left.-(r+\gamma z)^{5 / 3}{ }_{2} F_{1}\left[-\frac{5}{6}, \frac{1}{2}, 1 ; \frac{4 r \gamma z}{(r+\gamma z)^{2}}\right]\right\},
\end{gathered}
$$

where ${ }_{2} F_{1}$ denotes the Gauss hypergeometric function.

In the general case, the long-exposure Strehl ratio $S R$ is expressed through the four-times integral. However, if 
the residual structure function is isotropic, this expression is reduced to (Tatarski 1968)

$$
\begin{aligned}
S R= & \frac{16}{\pi} \int_{0}^{1} \mathrm{~d} \xi \xi\left(\arccos \xi-\xi \sqrt{1-\xi^{2}}\right) \\
& \exp \left[-\frac{1}{2} D_{\mathrm{S}_{\mathrm{R}}}(\xi D)\right],
\end{aligned}
$$

where $D$ is the telescope diameter.

As one can see from Eqs. $(5,6)$, one needs to know the $C_{n}^{2}$ profile to calculate the Strehl ratio of interest. In what follows we perform the calculations considering both the analytical Hufnagel model of $C_{n}^{2}$ profile Hufnagel (1974) and the experimental data obtained recently at SPM (Mexico).

\section{Calculation results with the Hufnagel and experimental $C_{n}^{2}$ profiles}

A useful model for $C_{n}^{2}$ profile corresponding to the nighttime observation conditions was suggested by Hufnagel (1974). This model is an analytical approximation of the experimental data, and it can be expressed as

$C_{n}^{2}(z)=C_{0}^{2}\left[\left(\frac{z}{z_{0}}\right)^{10} \exp \left\{-\frac{z}{z_{1}}\right\}+\exp \left\{-\frac{z}{z_{2}}\right\}\right]$,

where $C_{0}^{2}$ is the arbitrary scaling factor with dimension of length in the power $-2 / 3, z_{0}=4.63210^{3} \mathrm{~m}$, $z_{1}=10^{3} \mathrm{~m}$, and $z_{2}=1.510^{3} \mathrm{~m}$.

In the case of Hufnagel profile, the residual structure function is expressed analytically in terms of generalized hypergeometric functions. The final expression for $D_{\mathrm{S}_{\mathrm{R}}}$ can be obtained after substitution of Eq. (7) into Eq. (3) evaluating the integrals in the following sequence: over $\varphi, \theta, z, \kappa$. The intermediate integrals are evaluated applying the Mellin transformation technique, and the formulas needed for evaluations can be found in (Prudnikov et al. 1988).

The experimental $C_{n}^{2}$ profile used here is the median of 800 profiles obtained during three nights at the $2.1 \mathrm{~m}$ telescope of San Pedro Martir observatory, Baja California Mexico, in April 1997. The relevant profiles in an actual adaptive optics observation are the quasi-instantaneous ones, for the comparison of our results obtained with theoretical and experimental profiles, a median profile is better suited because it has a statistical value. A detailed description of this observing campaign will be presented in Avila et al. (1998). The measurements were accomplished with the generalized scidar (G-SCIDAR) which was suggested by Fuchs (1995) as a generalized version of the scidar technique originally proposed by Rocca et al. (1974). The data reduction consists in computing the spatial autocorrelation function of short exposure-time images of the scintillation pattern which is produced by a double star detected on a virtual plane a few kilometers beneath the pupil. A maximum entropy algorithm is used to retrieve

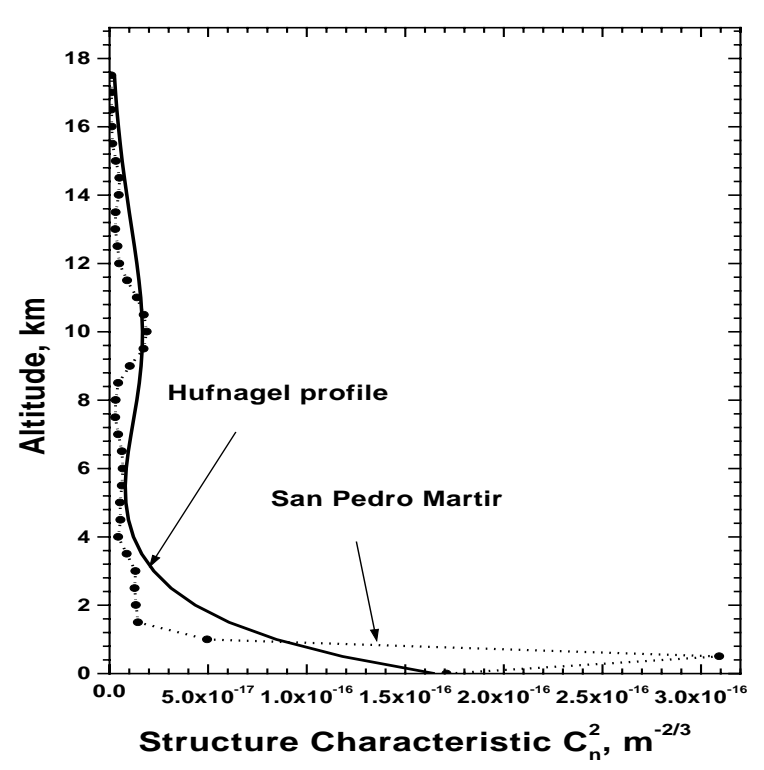

Fig. 1. Theoretical (Hufnagel) and experimental (San Pedro Mártir) $C_{n}^{2}$ profiles. The corresponding Fried parameter is equal to $16 \mathrm{~cm}$ (for wavelength $0.55 \mu \mathrm{m}$ ). The scaling factor for Hufnagel model has been chosen in such a way that the Fried parameters associated with two profiles be equal

the $C_{n}^{2}$ profile from the measured autocorrelation function. The experimental setup can be found in Avila et al. (1997), where the first implementation of the G-SCIDAR at a telescope focus is described.

Both the Hufnagel's and experimental profiles are plotted in Fig. 1. The scaling factor $C_{0}^{2}$ for Hufnagel profile has been chosen in such a way that the integrals $\int_{0}^{L} \mathrm{~d} z C_{n}^{2}(z)$ calculated for both profiles be equal. From the practical point of view it means that the same seeing conditions are considered for both cases.

Figures 2 and 3 plot the Strehl ratio calculated for both profiles versus the angular separation between the two stars. Figure 2 shows the ratio of interest for $2.1 \mathrm{~m}$ telescope which operates now in San Pedro Mártir observatory, while Fig. 3 presents the results for $6.5 \mathrm{~m}$ telescope which is planned for building in this observatory. Both graphs are plotted for three wavelengths which are of interest in astronomical applications: $0.55,1.25$ and $2.2 \mu \mathrm{m}$. Using these graphs, one can estimate the upper limit of the efficiency of off-axis adaptive correction.

As one can see from Figs. 2 and 3, despite of the seeing conditions associated with both profiles are equal, there is a significant difference in results to be obtained for off-axis adaptive correction. This difference appears because the profiles differ strongly in structure. The theoretical profile assumes a more or less smooth behavior of $C_{n}^{2}$ with altitude, while the experimental one has a pronounced layered structure with a great amount of the turbulence strength concentrated near to the ground. Since, as it follows from Eq. (5), the quality of correction is affected by the quantity 


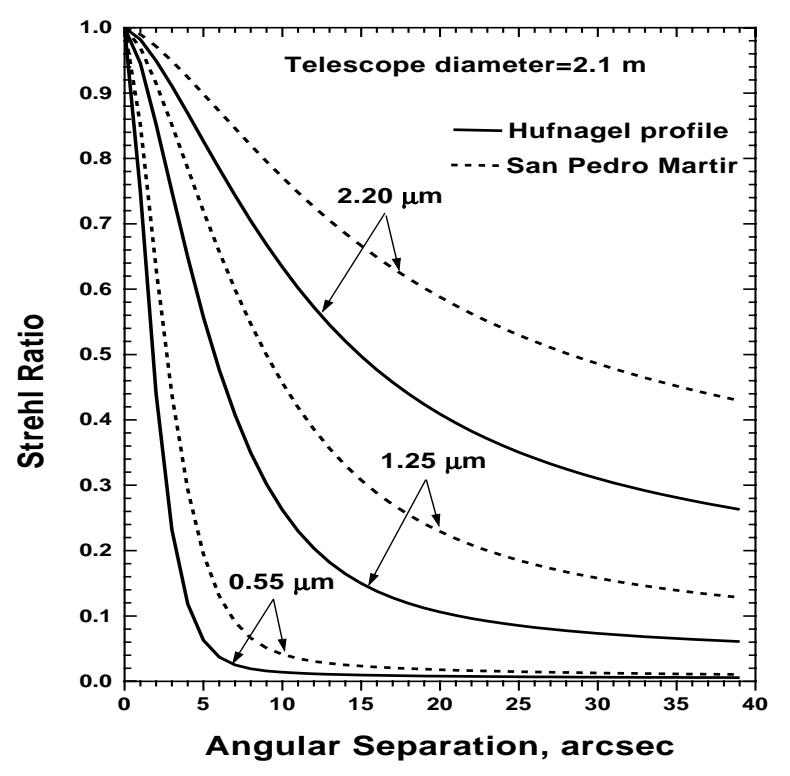

Fig. 2. Strehl ratio versus the angular separation between the stars for $2.1 \mathrm{~m}$ telescope

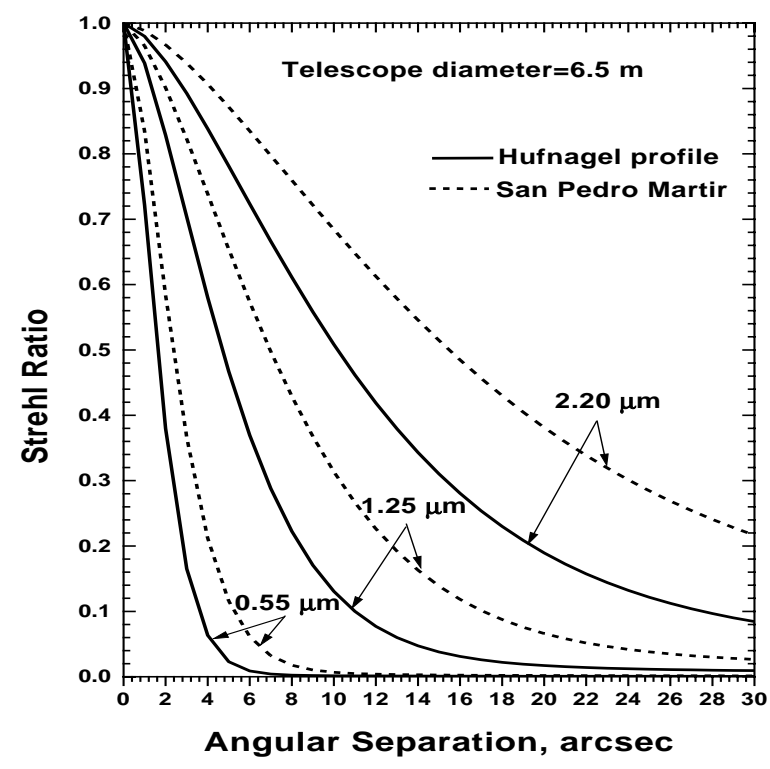

Fig. 3. Strehl ratio versus the angular separation between the stars for $6.5 \mathrm{~m}$ telescope

$\int_{0}^{L} \mathrm{~d} z z^{5 / 3} C_{n}^{2}(z)$, the profile with a turbulence strength concentrated mainly near-to-the ground gives better results for off-axis correction.
Basing on the results obtained, one may arrive to some conclusions in what concerns to the choice of the telescope location. On the one side, an off-axis adaptive correction is a promising approach which allows to override certain difficulties arising in observations of weak stars. However, the information which can be extracted from the conventional measurements of seeing conditions is not sufficient to predict the quality of such correction. So one needs to carry out some more advanced experiments allowing for the reconstruction of $C_{n}^{2}$ profile. Such measurements can be performed making use of different experimental techniques. We believe that the G-SCIDAR method is among the best candidates because it combines a good accuracy of profile reconstruction with a relatively low cost of measurements.

Acknowledgements. We are indebted to Dr. J. Vernin for kindly authorizing our use of the G-SCIDAR data in this investigation. This work was supported by Consejo Nacional de Ciencia y Tecnologia (Mexico) project 1020P-E9507 and by Sistema Nacional de Investigadores (Mexico).

\section{References}

Avila R., Vernin J., Masciadri E., 1997, Appl. Opt. 36, 7898 Avila R., Vernin J., Cuevas S., 1998 (submitted to PASP)

Chassat F., 1989, J. Opt. (Paris) 20, 13

Fried D.L., 1978, J. Opt. Soc. Am. 68, 1651

Fried D.L., 1982, J. Opt. Soc. Am. 72, 52

Fuchs A., 1995, Ph.D. dissertation. Université de Nice-Sophia Antipolis

Hufnagel R.E., 1974, in: Optical Propagation through Turbulence, OSA Technical Digest Series, OSA, Washington DC, p. WA1-1-WA1-4

Prudnikov A.P., Brychkov Yu.A., Marichev O.I., 1988, Integrals and Series. Gordon \& Breach, New York, Vol. 2, 3

Rocca A., Roddier F., Vernin J., 1974, J. Opt. Soc. Am. A 64, 1000

Roddier F., 1981, Progr. Opt. 19, 283

Sasiela R.J., Shelton J.D., 1993, J. Opt. Soc. Am. A 10, 646

Shapiro J.H., 1976, J. Opt. Soc. Am. 66, 469

Tatarski V.I., 1968, The Effects of the Turbulent Atmosphere on Wave Propagation, National Science Foundation Report TT-68-50464

Vitrichenko E.A., Voitsekhovich V.V., Mishchenko M.I., 1984, News of Academy of Sciences USSR: Atmospheric and Oceanic Physics 20, No. 9, p. 870

Wang C.P., 1975, Opt. Comm. 14, 200

Welsh B.M., Gardner C.S., 1991, J. Opt. Soc. Am. A 8, 69 